EOmmun: Communication et organisation

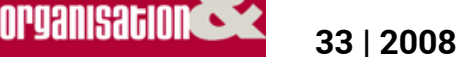

Conduire le changement organisationnel ?

\title{
Entretien avec Pierre Collerette
}

Elizabeth Gardère et Pierre Collerette

\section{OpenEdition}

Journals

Édition électronique

URL : http://journals.openedition.org/communicationorganisation/538

DOI : 10.4000/communicationorganisation.538

ISSN : $1775-3546$

Éditeur

Presses universitaires de Bordeaux

Édition imprimée

Date de publication : 1 juin 2008

Pagination : 145-149

ISBN : 978-2-86781-506-5

ISSN : 1168-5549

Référence électronique

Elizabeth Gardère et Pierre Collerette, "Entretien avec Pierre Collerette », Communication et organisation [En ligne], 33 | 2008, mis en ligne le 01 juin 2011, consulté le 15 septembre 2020. URL http://journals.openedition.org/communicationorganisation/538

(c) Presses universitaires de Bordeaux 


\title{
Entretien avec Pierre Collerette
}

\author{
par Elizabeth Gardère
}

pierre.collerette@uqo.ca

elizabeth.gardere@u-bordeaux1.fr

\begin{abstract}
Pierre Collerette est professeur en gestion à l'Université du Québec en Outaouais et consultant pour de nombreux projets de changement et de réorganisation au Canada et à l'étranger. Sa double formation en psychologie des relations humaines et en sciences de l'information et de la communication donne matière à une lecture interdisciplinaire de la problématique du numéro 33 de la revue Communication \& Organisation.
\end{abstract}

Entre 2001 et 2007, il consacre sept articles au changement organisationnel initialement publiés dans la Revue ISO Management System, avant d'être mis en ligne (http://w3.uqo.ca/collpi01/).

Le cinquième volet est consacré à l'axe Communication et changement - (http://w3.uqo.ca/collpi01/fr/gest_chang_part5.pdf).

Auteur de deux ouvrages complémentaires sur la question ${ }^{1}$, il nous livre quelques pistes pour nourrir la réflexion sur la gestion du changement organisationnel.

Pierre Collerette, de nombreux travaux sont consacrés au changement organisationnel. Pour s'inscrire dans l'approche communicationnelle de la revue Communication \& Organisation, quelle définition pouvez-vous en donner? Quelles ouvertures laisset-elle aux chercheurs en sciences de l'information et de la communication?

1 Collerette P., Delisle G., Perron., (1998), Le changement organisationnel. Théorie et pratique, Presses de l'Université du Québec. 173 p.

Collerette P., Schneider R., (1996), Le pilotage du changement. Une approche stratégique et pratique, Presses de l'Université du Québec. $365 \mathrm{p}$. 


\section{Entretien}

Pour moi, il y a changement organisationnel lorsque les comportements et pratiques des acteurs d'une organisation ont été modifiés de façon relativement durable. Cette définition ne réfère pas directement à l'approche communicationnelle, mais dans la plupart des cas elle suppose qu'il y ait des échanges entre les acteurs pour mener à une certaine acceptation collective des nouvelles pratiques. Les processus communicationnels seraient donc critiques dans le processus d'adoption des changements organisationnels, et il y a là pour les chercheurs un vaste terrain à explorer, notamment pour mettre en lumière les pratiques de gestion du changement qui en conditionnent l'adoption ou le refus.

\section{Quels sont les enjeux de la communication face au changement?}

Les gestionnaires de changement ont souvent tendance à concentrer leurs efforts sur le contenu de l'information et sur les modalités de sa transmission, alors que les principaux enjeux se situent probablement au niveau de la relation, qui joue un grand rôle dans l'interprétation des messages. Ce qui est en cause, c'est la capacité des promoteurs du changement d'établir une sorte de relation dialogique avec les destinataires, visant à une compréhension partagée des problèmes, des défis et des solutions. Bref, une communication mise au service d'une construction partagée du sens.

Les médias de communication jouent un rôle important dans l'accompagnement du changement. Que dire des conduites de communication?

Les médias ont l'avantage d'être faciles d'accès et d'atteindre de larges auditoires, mais pour autant cela ne les rend pas efficaces à des fins de changement. J'observe que dans plusieurs cas, la communication par des médias formels est réinterprétée dans les canaux informels sur la base d'autres critères. Ce qui devient alors déterminant, c'est la relation avec les leaders du changement, et plus spécialement la cohérence entre leurs conduites et leur discours. Bref, la nature de la relation aurait préséance sur l'information, et de surcroît sur les médias. En mettant l'accent sur des dispositifs techniques, on risque de négliger l'essentiel. D'ailleurs, les recherches visant à départager les médias pauvres des médias riches montrent que ces derniers impliquent de la proximité entre les interlocuteurs, qui leur permet de prendre en compte les conduites. En fait, toutes les conduites du management ont une valeur communicationnelle et leur impact est sans doute plus marquant que quelque autre médium. 
Vos ouvrages convoquent l'analyse systémique. En quoi ce modèle offre-t-il une grille de référence pour une perception cohérente des environnements à l'intérieur desquels nous agissons?

En posant que les systèmes sont des entités dynamiques, l'analyse systémique fait du changement un phénomène normal, alimenté par des forces naturelles, et dès lors propose des repères permettant de mieux en saisir la genèse. Elle permet du même coup d'aborder la problématique du changement avec un état d'esprit positif plutôt que défensif. Le changement n'y fait pas figure de drame ou d'incongruité, mais devient un événement probable et influençable. Elle a aussi le mérite de montrer que non seulement plusieurs facteurs participent aux situations, mais aussi qu'un changement peut modifier l'équilibre du système, de sorte que des stratégies trop simples seront inappropriées.

Une taxonomie des approches du changement offre le choix pour introduire le changement dans l'organisation. On parlera, entre autres, d'approches stratégiques ou tactiques, de typologies en fonction de l'agent, de la dimension du pouvoir. Selon quels critères ce choix s'opère-t-il ?

Une taxonomie ouvre une fenêtre sur un aspect de la réalité, mais celle-ci est composite et on ne peut prétendre en saisir l'essence en l'abordant sous un seul angle. Aussi, pour saisir à la fois les enjeux à surveiller et les leviers pouvant être activés, l'intervenant gagne à diversifier ses angles d'analyse plutôt que de chercher quelle taxonomie privilégier. La diversité des angles d'analyse ajoute de l'éclairage aux situations et enrichit la gamme des options auxquelles on peut recourir pour à la fois accroître les chances de succès, et minimiser les effets négatifs sur les acteurs internes et externes. À la limite, s'il fallait donner préséance à une taxonomie, je pense qu'il faudrait choisir celle qui éclaire le mieux la réalité perçue par les destinataires du changement.

Le processus du changement et ses implications pour l'agent sont des éléments clefs d'une réalité organisationnelle en mutation. Comment identifier les éléments déclencheurs et accompagner la démarche?

Je me limiterai à mentionner que souvent les plus puissants déclencheurs sont liés aux problèmes que les gens vivent dans leur quotidien ou ceux se profilant à l'horizon. Les promoteurs du changement devraient donc s'employer à arrimer leurs intentions de changement à ces difficultés, qui viendront donner un sens aux efforts 


\section{Entretien}

demandés. Souvent, on propose aux destinataires des visions désincarnées de leur réalité concrète, alors que l'on pourrait y trouver des lacunes pour lesquelles le sens d'une action correctrice émergerait presque naturellement. Pour que ces déclencheurs suscitent une mobilisation, il faut par ailleurs que ces problèmes soient discutés ouvertement en vue de parvenir à une lecture partagée. Ce n'est pas suffisant pour un accompagnement du changement, mais c'est, je pense, une condition importante pour l'appropriation du changement, sans quoi on se lance dans des opérations purement mécaniques où l'engagement des gens fait défaut.

Les attitudes et les valeurs évoluent avec le changement. Quelles sont les tendances qui se dégagent?

Certains proposent d'axer les interventions sur les valeurs et les attitudes des gens, en faisant valoir qu'elles constituent les racines des comportements. Je suis devenu très nuancé avec cette conception. Les attitudes indiquent une prédisposition à agir d'une façon donnée, mais ne sont pas nécessairement des prédicteurs fiables du comportement, qui est aussi influencé par des facteurs situationnels, dont la pression des pairs. En outre, malgré leur caractère collectif, les attitudes et les valeurs relèvent de l'intimité des individus, ce qui en rend l'accès très difficile pour l'intervenant. Dans un certain nombre de cas, on peut mettre l'accent davantage sur les pratiques, qui à leur tour vont influencer les attitudes et les valeurs, surtout lorsque ces pratiques ont peu de résonance valorielle. J'ajouterai que l'on confond parfois les changements de valeurs et d'habitudes; certains changements présentés comme culturels, modifient en fait des habitudes bien ancrées, dont la composante normative est faible.

Quelle est la position des acteurs dans l'entreprise en changement? Quels sont les enjeux du changement chez l'individu?

L'individu doit composer avec au moins trois grandes sources de tension. La plus importante peut-être, c'est la pression des pairs significatifs par rapport à la position qu'il est encouragé à prendre à l'endroit du changement. N'oublions pas que le changement organisationnel s'opère sur une scène sociale qui encadre la marge de manœuvre individuelle. Une autre source de tension provient des modifications qu'il doit apporter à ses rituels, avec parfois des difficultés d'ordre cognitif ou valoriel. Une troisième source découle des efforts requis pour apprendre et maîtriser les nouvelles compétences, qui du reste ne sont parfois pas au point. Ces sources de tension s'enchevêtrent et s'alimentent, accroissant la vulnérabilité des 
gens, d'où l'importance d'un accompagnement soutenu des destinataires du changement.

Le changement organisationnel se fait au prix de résistances. La préparation au changement et l'analyse préalable pour lever les objections ne sont pas toujours suffisantes pour anticiper les obstacles. Quelles sont les précautions à prendre? Quelle place donner à la communication?

Pour prévenir la montée en force des résistances, il est important de se consacrer à un travail d'éveil dont l'intention est d'en arriver à une reconnaissance des motifs justifiant le changement et des solutions les mieux adaptées. Une communication riche et soutenue devient alors une condition de réussite incontournable. Il faut bien sûr rester réaliste et savoir que dans certaines situations les intérêts des parties prenantes ne peuvent converger. Dans certains de ces cas, on peut néanmoins minimiser les résistances grâce à un accompagnement rigoureux lors de la mise en œuvre. Ainsi, à défaut d'avoir rallié les destinataires à l'idée du changement, on peut quand même limiter les sources de tension, et faciliter d'autant leur adaptation au changement.

\section{Que souhaiteriez vous rajouter?}

En matière de communication du changement on met souvent l'accent sur l'emballage de l'information et sa transmission, avec le risque de négliger des aspects plus conséquents, comme la relation avec les destinataires. Sans une relation suffisamment coopérative, les approches axées sur la transmission de l'information seront rarement suffisantes pour créer des conditions favorables à la réussite du changement. C'est pourquoi les gestionnaires de changement doivent prévoir du temps pour discuter avec les destinataires car leur disposition à l'endroit du changement en sera influencée. 